\title{
SCIDiC
}

International Journal of Behavioral Research \& Psychology (IJBRP)

ISSN 2332-3000

\section{Continued Focus on Sleep Deprivation Effects on Health Necessary}

Editorial

Sexton-Radek $\mathrm{K}^{*}$

Professor, Elmhurst College, Suburban Pulmonary \& Sleep Associates, USA.

The scientific study of sleep deprivation, while longstanding, continues to be needed as the findings directly impact public health concerns. Increased symptomology in medical conditions, development of symptoms of disease such as increased blood pressure and heart rate, heightened blood sugar levels, increased infections due to reduced immune functioning, and inflammation [4]. In addition to the medical consequences of sleep deprivation are the reductions in psychological functioning. Decreased attention and focus have been consistently found with products of this function measured in sleep deprived college student low grades and decreased functioning in the classroom process [8]. The micro changes in cortical functioning in terms of reduced hippocampal synaptical activity to the cortex resulting in reduced processing and memory functioning [5]. Impaired synaptic functioning also leads to impaired synaptic plasticity. It is believed that this impaired cellular functioning is the root of the poor communication ability leading to compromised cortex driven activities of attention, concentration and memory [1].

Researchers examining the effects of sleep deprivation have identified distinct physiological effects of acute and chronic sleep deprivation $[4,6]$. Both acute and chronic sleep deprivation effects, however, involve decreases in cognitive functioning of reduced alertness, attention, slowed responses, attentional lapses and wake-state instability [6]. Chronic sleep deprivation begins to attrite the motor functioning capacity of the sleeper with 24 hours of sleep loss. Loss of motor functioning is followed by changes in self-perception of motor capabilities $[6,7]$. The sleep deprived individual becomes unaware of the degree of their incapacity. Measurements of decreased response time in sleep deprived individuals has been found $[9,10]$.

With the empirical results of sleep deprivation on behavior, extended investigation is needed. The effects both short and long term of sleep deprivation are unknown. The process by which physiological and consequential behavioral deficits emerge is not identified. The mechanism of cellular change leading to hypotheses cortically driven behaviors needs further investigation. Such continued focus will help to lead to directions and pathways for recovery for individuals with sleep deprivation. Ideally, specific findings such as the measured results of improved sleep quality in individuals with caffeine reduction, for example would enhance our understanding of both process and outcome of sleep deprivation on behavior [2]. Further, perhaps sleep schedule and sleep-wake style such as chronotypes of morningness versus eveningness would enrich the understanding of the wakefulness patterns and functioning [3]. In all, further research to enhance understanding, substantiate neuroscience findings to a pathway level of analysis and to design applications for recovery from sleep deprivation are essential.

\section{References}

[1]. Alhola P, Polo-Kantola P. Sleep deprivation: Impact on cognitive performance. Neuropsychiatric Disease and Treatment. 2007; 3(5): 553-567. PMID: 19300585.

[2]. Anderson C, Horne J. Placebo response to caffeine improves reaction time performance in sleepy people. Human Psychopharmacology: Clinical \& Experimental. 2008; 23: 333-336. Doi:10.1002/hup.931. PMID: 18350573.

[3]. Barclay NL, Myachykov A. Sustained wakefulness and visual attention: moderation by chronotype. Experimental Brain Research. 2017; 235: 57-68. PMID: 27624836. Doi: 10.1007/s00221-016-4772-8.

[4]. Carskadon MA. Sleep Deprivation: Health consequences and societal impact. Medical Clinics of North America. 2004; 88:767-776. PMID: 15087215. Doi: 10.1016/j.mcna2004.03.001

[5]. Chee ML, Chuah LY. Functional neuroimaging insights into how sleep and sleep deprivation affect memory and cognition. Current Opinion in Neurology. 2008; 21:417-423. PMID: 18607201.

[6]. Daviaux Y, Mignardot JB, Cornu C, Deschamps T. Effects of total sleep deprivation on the perception of action capabilities. Experimental Brain Research. 2014; 232:2243-2253. PMID: 24691757. Doi: 10.1007/s00221-014-3915-z.

[7]. Lisper HO, Kjellberg A. Effects of 24-hour Sleep Deprivation on rate of decrement in a 10-minute auditory reaction time task. Journal of Experimental Psychology. 1972; 96(2): 287-290. PMID: 4645958.

[8]. Stepan ME, Altmann EM, Fenn KM. Effects of total sleep deprivation on procedural placekeeping: More than just lapses of attention. Journal of Experimental Psychology: General. 2019. PMID: 31750712. Dioi: 10.1037/ xge0000717.

[9]. vandenBerg J, Neely G. Performance on simple reaction time task while sleep-deprived. Perceptual and Motor Skills. 2006; 102: 589-599. PMID: 16826680. Doi: $10.2466 /$ pms.102.2.589-599.

[10]. Wlodarczyk D, Jaskowski P, Nowik A. Influence of sleep deprivation and auditory intensity on reaction time and response force. Perceptual and Motor Skills. 2002; 94: 1101-1112. PMID: 12186231.

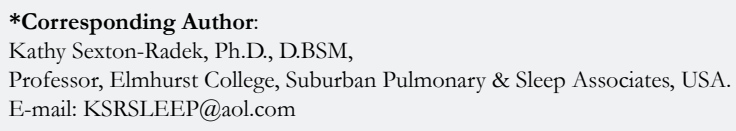

Copyright: Sexton-Radek $\mathbf{K}^{\circ}$ 2019. This is an open-access article distributed under the terms of the Creative Commons Attribution License, which permits unrestricted use, distribution and reproduction in any medium, provided the original author and source are credited. 